\title{
Alcohol outlet density and alcohol consumption in Los Angeles county and southern Louisiana
}

Matthias Schonlau ${ }^{1}$, Richard Scribner ${ }^{2}$, Thomas A. Farley ${ }^{3}$, Katherine P. Theall ${ }^{2}$, Ricky N. Bluthenthal ${ }^{1,4}$, Molly Scott ${ }^{1}$, Deborah A. Cohen ${ }^{1}$

${ }^{1}$ RAND Corporation, 4570 Fifth Avenue, Suite 600, Pittsburgh, PA 15213, USA; ${ }^{2}$ Louisiana State University, Health Sciences Center, School of Public Health, 600 Lakeshore Drive, Suite 200, New Orleans, LA 70122, USA; ${ }^{3}$ Tulane University, School of Public Health and Tropical Medicine, Department of Community Health Sciences, 1440 Canal Street, Suite 2301, New Orleans, LA 70112, USA; ${ }^{4}$ Urban Community Research Center, Sociology Department, California State University, Dominguez Hills, 1000 East Victoria Street, Carson, CA 90747, USA

\begin{abstract}
The objective of this study was to assess the relationship between alcohol availability, as measured by the density of off-premise alcohol outlets, and alcohol consumption in Los Angeles county and southern Louisiana, USA. Consumption information was collected through a telephone survey of 2,881 households in Los Angeles county and pre-Katrina southern Louisiana, nested within 220 census tracts. Respondents' addresses were geo-coded and both neighbourhood (census tracts and buffers of varying sizes) and individual (network distance to the closest alcohol outlet) estimates of off-sale alcohol outlet density were computed. Alcohol outlet density was not associated with the percentage of people who were drinkers in either site. Alcohol outlet density was associated with the quantity of consumption among drinkers in Louisiana but not in Los Angeles. Outlet density within a one-mile buffer of the respondent's home was more strongly associated with alcohol consumption than outlet density in the respondent's census tract. The conclusion is that the relationship between neighbourhood alcohol outlet density and alcohol consumption is complex and may vary due to differences in neighbourhood design and travel patterns.
\end{abstract}

Keywords: alcohol outlet density, alcohol consumption, Los Angeles county, southern Louisiana.

\section{Introduction}

Alcohol use and abuse and their consequences are significant problems in the United States. The Center for Disease Control and Prevention (CDC) estimates $61 \%$ of adults drink alcohol (http://www.cdc.gov/nchs/fastats/alcohol.htm) and $32 \%$ of current drinkers had five or more drinks on at least one day in the past year. The availability of alcohol has been associated with drinking and driv-

Corresponding author:

Matthias Schonlau

RAND Corporation

4570 Fifth Avenue, Suite 600

Pittsburgh, PA 15213, USA

Tel. +1 412683 2300/4917; Fax +1 4126832800

E-mail: matt@rand.org ing (Treno et al., 2003), injury (Treno et al., 2001), motor vehicle crashes (Scribner et al., 1994), homicide (Scribner et al., 1999), and greater rates of assault (Scribner et al., 1995; Lipton and Gruenewald, 2002). There are over 20,000 alcoholattributed deaths and additional 12,000 deaths due to alcoholic liver disease yearly (http://www.cdc.gov/nchs/fastats/alcohol.htm).

The availability of alcohol has also been linked with increased alcohol consumption. Surveying employees in a manufacturing plant, Ames and Grube (1999) found that alcohol availability at work was associated with greater consumption. Abbey et al. (1990) found that distance to the closest alcohol outlet was indirectly associated with alcohol consumption through perceived convenience of buying 
alcohol and subjective measures. In a sample of college students covering a wide range of outlet densities Weitzman et al. (2003) found that alcohol outlet density correlated with heavy drinking, frequent drinking and drinking-related problems. A truncated range of outlet densities might make it harder to observe effects related to outlet density.

The majority of previous studies have utilized aggregate measures of alcohol consumption and alcohol availability exposure, for example, at the ZIP code or census tract level. Aggregate or ecological analyses do not control for individual demographic differences (Greenland, 2001) and inferences from aggregate data about individual behaviours can be misleading. This is known as the ecological fallacy (Robinson, 1950). Some recent studies of the relationship between alcohol availability and either consumption or alcohol-related health outcomes have combined characteristics at the individual and aggregate level in multilevel or hierarchical analyses.

The present study examined whether alcohol availability was a determinant of alcohol consumption across more than 200 neighbourhoods in two distinct geographical areas. Because we used individual-level data, we avoided ecological bias. This study was designed to add to the existing literature in the following ways. Firstly, we investigated measures of the quantity and frequency of ethanol consumption separately in two steps: we first investigated the association of availability and drinking status (drinker versus non-drinker) and then estimated the association with ethanol consumption among drinkers only. Secondly, we further explored whether the quantity and frequency of drinking was more strongly associated with neighbourhood exposures measured using census tract boundaries compared to buffers of varying distances from an individual's home.

\section{Materials and methods}

Data for these analyses come from the cross-sectional study of alcohol availability, marketing, pro- motion and consumption conducted in 220 census tracts in Los Angeles county and pre-Katrina southern Louisiana (106 in Louisiana and 114 in Los Angeles) (see also Bluthenthal et al., 2008; Scott et al., 2008; Theall et al., 2009). Data from the crosssectional a household level telephone survey were combined with data on the number of alcohol outlets to examine the hypothesized relationships. The phone survey was conducted from 4 October 2004 to 28 August 2005 in Louisiana and from 4 October 2004 to 19 October 2005 in Los Angeles county.

The sample frame consisted of urban census tracts in 26 contiguous parishes in southeastern Louisiana and within 20 miles of Charles R. Drew University of Medicine and Science in Los Angeles county. A radius of 20 miles - while opportunistic - covers most of the census tracts in Los Angeles. An urban census tract was defined to contain at least 2,000 persons per square mile. The U.S. census bureau considers a census block group urban if they have a population density of at least 1,000 persons per square mile and are adjacent to another census block group with a population density of at least 500. We drew a random sample of urban census tracts stratified by site.

\section{Consumption survey}

Within each sampled census tract we conducted a survey of households with listed telephone numbers to ask questions about various health behaviours, with particular emphasis on alcohol consumption. Households were sampled using a systematic sample of a household list provided by a commercial vendor to yield approximately 10 households per census tract. A list-based sample was chosen because, in addition to the phone numbers, it provided access to household addresses needed for geo-coding. The respondent within each sampled household was selected using the most-recent-birthday method. Up to 25 contact attempts were made before a household was removed from the sample. Procedures were approved by the institutional review board of 
RAND. The questionnaire contained 84 questions but, due to skip patterns, it took only $15-20 \mathrm{~min}$ to complete it. Advance letters were sent to all unique household addresses and a toll free phone number was provided if the respondent wanted to initiate the survey. Participants were informed that they would be sent a check of US\$ 15 upon completion of the survey.

We computed two consumption measures based on the survey, i.e. the average daily ethanol consumption based on the previous 12 months, and one based on the previous 90 days. The average daily ethanol consumption based on the previous 12 months was defined as:

$$
\text { ethanol }_{12 \text { months }}=\text { drinkdays } * \text { drinks } * 0.6 / 365
$$

where drinkdays $=$ the number of drinking days per year; drinks = the number of drinks per occasion; and, 0.6 = the amount of ethanol in an average drink (Kerr et al., 2005). The number of drinkdays was computed from a question about drinking frequency with the levels "at least once a day", "nearly every day", "three or four times a week", "once or twice a week", "two or three times a month", "about once a month", "less once a month but at least once a year" and "none in the last 12 months". The respondent was then asked how many drinks he/she usually had on those days when he/she drank alcohol in the last 12 months. This 12 -month measure is a quantity/frequency measure multiplied by a constant $(0.6 / 365)$.

The average daily ethanol consumption based on the previous 90 days was computed as:

$$
\text { ethanol }{ }_{90 \text { days }}=\text { ethanol } * \text { ounces } * \text { drinks } * \text { drinkdays }
$$

where ethanol = the alcohol content of the respondent's most common drink in the last 90 days; ounces = the size of that drink in ounces, drinks the number of drinks per occasion; and, drinkdays = the number of days drinking within the last 90 days. The number of drinking days was computed from a question about drinking frequency with the cate- gories, "never", "less than once a month", "about once a month", "a few times a month", "about once a week", "several times a week", "once a day" and "several times a day". We asked about the brand name of the beverage the respondent drank most often in the previous 90 days, the type of beverage (beer, wine and eight other categories), the specific type of container the respondent consumed this drink in, and how many ounces this type of container holds. The type of beverage was used to compute ethanol per ounce.

\section{Alcohol availability measurement}

Addresses of alcohol outlets were obtained from the California Department of Alcohol Beverage Control $(\mathrm{ABC})$ and the Louisiana Department of Alcohol and Tobacco Control (ATC). The addresses were geo-coded. We included in the analysis all outlets where alcohol purchased was for consumption off-premise. We restricted the analysis to offpremise outlets because of their association with problem drinking and community level morbidity and mortality. We measured alcohol availability through the number of nearby alcohol outlets in several ways:

(i) network distance to the nearest outlet;

(ii) number of outlets in the census tract in which the respondent lived; and

(iii) number of outlets in buffers around respondents' homes.

We geo-coded the respondent's home and computed the number of alcohol outlets within buffers of $0.1,0.25,0.5$ and 1.0 mile radii. We chose these distances near half a mile because half a mile represents a $10-15$ min walk which is considered a maximum walking distance for some people (Lee et al., 2003; Truong and Sturm, 2003); we chose not to normalize the number of outlets by dividing by the number of roadway miles, square miles, or population size. In densely populated areas such a definition might have resulted in a low density even when the individual lives very close to an alcohol outlet. 


\section{Missing values}

The number of missing values was under $10 \%$ for all variables except for the brand name of the alcohol drink (45.8\%). Therefore we imputed missing values multiple times to capture the variation between imputations. We imputed missing values five times using a Markov chain Monte Carlo algorithm (Schafer, 1997). For indicator variables the input values were stochastically assigned 0 or 1 using Bernoulli sampling. The entries rely on the usual missing at random assumption (MAR). The use of the MAR assumption is unverifiable but standard practice; we have found no reason to discredit this assumption. We used Rubin's formula (Rubin, 1987) to combine the regression results from different imputations.

\section{Statistical analyses}

Because the study was conducted in two very different geographical locations, we conducted all analyses separately by study location. To examine how availability as measured by the number of outlets relates to alcohol consumption, we first explored descriptive statistics on the number of outlets within census tracts as well as within radii of $0.1,0.25,0.5$ and 1 mile around survey respondents' homes and identified which radius had the largest association with consumption.

We divided alcohol consumption into two components: (i) whether or not the respondent was a drinker and (ii) if the respondent was a drinker, how much alcohol the respondent consumed. We adjusted for gender, age, race/ethnicity and self-reported income as they may be associated with consumption. Because education was correlated with income it was omitted as a covariate.

For the first component, we regressed an indicator variable of drinking status (drinker/non-drinker) on covariates, for the second component we regressed each of the two measures of ethanol consumption on covariates. We used log-transformed ethanol measurements. Because log values are harder to interpret, we used the model to compute sampleaveraged predictions of daily ethanol consumption of two slightly altered data sets for each variable. Specifically, for indicator variables we computed average predictions with the indicator (i) set to zero and (ii) set to one in the entire sample; for continuous variables we set the variable to the $25^{\text {th }}$ and $75^{\text {th }}$ percentiles, respectively.

To account for possible correlation within tracts (e.g. due to overlapping buffers) we also explored a random intercept hierarchical model and a spatial regression using a power exponential correlation function within tract. Because tracts were not necessarily contiguous due to random sampling, the spatial regression also assumed that measurements for respondents in different tracts were uncorrelated.

For the hierarchical regression of ethanol consumption we computed the intraclass cluster correlation (ICC) to assess what fraction of the variation in consumption occurs at the census tract level. The ICC for a hierarchical logistic regression is difficult to interpret because the distribution at the individual level is binomial and the distribution and the census tract level is normal. The variance at the individual level is a function of prevalence. Instead, we translated the census tract level variance into a median odds ratio (MOR) (Merlo et al., 2006). The MOR is a measure of variability and can be interpreted as the median increased risk in becoming a drinker by moving from one census tract to another (the odds ratio (OR) is always formed as an increase, i.e. as a value greater than 1 ; for differences, this is analogous to considering absolute differences rather than differences). In the absence of a census tract-level effect the risk would be the same in all census tracts.

We used the Hosmer Lemeshow goodness of fit test and Pregibon's link test (Pregibon, 1980) to assess model fit. We also examined residual plots and variance inflation factor to check for multicollinearity among independent variables. 
We used SAS version 9.1.3 for imputations, spatial autocorrelation models, and for hierarchical logistic regressions. All other analyses were conducted in STATA version 9.

\section{Results}

\section{Survey results}

Data were collected in 220 census tracts, namely 114 from Los Angeles county and 106 in southeastern Louisiana. Data collection in Louisiana was halted early due to Hurricane Katrina, resulting in a loss of data from eight additional census tracts. The cooperation rate, the proportion of all cases interviewed of all eligible respondents ever contacted was $76.2 \%$ in Los Angeles and $79.8 \%$ in Louisiana for the phone survey. The response rate, RR3 (AAPOR, 2006), which also takes into account phone numbers we were unable to reach to verify eligibility, was $34.4 \%$ in Los Angeles and $37.9 \%$ in southern Louisiana. Response rates of this magnitude have become common even among government surveys. For example, the 2005 behavioural risk factor surveillance system (BRFSS) survey's response rates were $29.2 \%$ in California and $36.5 \%$ in Louisiana (http://aspe.hhs.gov/hsp/06/Catalog-AI-ANNA/BRFSS.htm). The number of phone survey respondents was 1,578 in Los Angeles and 1,303 in Louisiana. In Louisiana there was an average of 13.8 respondents per census tract. In Los Angeles there was an average of 12.3 respondents per census tract.
Table 1 gives sample characteristics by state. Approximately two-third of respondents were female. The samples differed with respect to race and ethnicity, with the Louisiana sample including approximately equal numbers of whites and African-Americans, and the Los Angeles sample more than one third Hispanics. Separate analyses were conducted by state and adjusted for the sample characteristics.

The mean number of off-premise alcohol outlets in buffers of various radii is shown in Table 2. The mean number of alcohol outlets ranged from approximately 0.25 outlets in a 0.1 mile radius to 20 in a 1 mile radius. Census tracts contained 3.3 outlets on average, corresponding in size to a buffer zone with a radius between a 0.25 mile and a 0.5

Table 1. Sample demographic characteristics by state.

\begin{tabular}{lcc}
\hline Variable & Louisiana & Los Angeles \\
\hline Age (mean) & 44.1 & 41.9 \\
Age (standard deviation) & 13.0 & 13.3 \\
Gender & & \\
$\quad$ Male (\%) & 33.0 & 38.6 \\
Female (\%) & 67.0 & 61.4 \\
Respondent's income (\%) & & \\
$\quad$ <US\$ 15,000 & 24.3 & 22.9 \\
$\quad$ US\$ 15,000 - 25,000 & 16.3 & 18.8 \\
US\$ 25,000 - 50,000 & 24.6 & 21.4 \\
$\quad$ US\$ 50,000 - 75,000 & 14.2 & 13.3 \\
>US\$ 75,000 & 20.6 & 23.6 \\
Race/ethnicity & & \\
$\quad$ Non-Hispanic white (\%) & 50.4 & 35.1 \\
$\quad$ Non-Hispanic black (\%) & 42.1 & 16.3 \\
Other (\%) & 1.9 & 4.1 \\
Asian (\%) & 1.0 & 5.5 \\
$\quad$ Hispanic (\%) & 4.6 & 38.9 \\
\hline
\end{tabular}

Table 2. Descriptive statistics of the number of alcohol outlets in buffers of varying radii around respondents, in census tract and mean distance to closest alcohol outlet by study site.

\begin{tabular}{|c|c|c|c|c|c|c|c|c|c|c|}
\hline \multirow[b]{2}{*}{ No. of outlets in } & \multicolumn{5}{|c|}{ Louisiana } & \multicolumn{5}{|c|}{ Los Angeles } \\
\hline & Min & $\begin{array}{l}1 \text { st } \\
\text { quartile }\end{array}$ & Mean & $\begin{array}{l}\text { 3rd } \\
\text { quartile }\end{array}$ & $\operatorname{Max}$ & Min & $\begin{array}{l}\text { 1st } \\
\text { quartile }\end{array}$ & Mean & $\begin{array}{l}\text { 3rd } \\
\text { quartile }\end{array}$ & Max \\
\hline 0.1-mile buffer & 0 & 0 & 0.19 & 0 & 4 & 0 & 0 & 0.17 & 0 & 4 \\
\hline 0.25 -mile buffer & 0 & 0 & 1.33 & 2 & 16 & 0 & 0 & 1.27 & 2 & 8 \\
\hline 0.5 -mile buffer & 0 & 0 & 5.41 & 8 & 43 & 0 & 2 & 5.30 & 8 & 19 \\
\hline 1.0-mile buffer & 0 & 6 & 10.58 & 27 & 115 & 0 & 10 & 19.67 & 28 & 63 \\
\hline Census tract & 1 & 2 & 3.30 & 5 & 14 & 1 & 2 & 3.36 & 5 & 9 \\
\hline Distance to closest outlet (miles) & 0 & 0.36 & 0.67 & 0.62 & 30.84 & 0 & 0.17 & 0.48 & 0.49 & 4.50 \\
\hline
\end{tabular}


mile. The mean numbers of outlets in various categories in Los Angeles and Louisiana were comparable and not significantly different. However, the mean distance to the closest outlet in Louisiana was somewhat larger and the difference showed statistical significance.

\section{Drinkers versus non-drinkers}

The MOR is a measure of how much drinking status varies by census tract. The smallest MOR, i.e. 1, implies no variability in drinking status among census tracts; a large MOR implies a large variability. The unadjusted MOR for drinking status were 1.77 $(\mathrm{P}=0.002)$ for Louisiana and $1.74(\mathrm{P}<0.001)$ for Los Angeles. This means that the median risk in becoming a drinker by moving from one census tract to another increased by $77 \%$ in Louisiana and

Table 3. Adjusted log ORs of drinking status (yes/no) for covariates separately for Louisiana and Los Angeles.

\begin{tabular}{lrrrr}
\hline & \multicolumn{2}{c}{ Louisiana } & \multicolumn{2}{c}{ Los Angeles } \\
\cline { 2 - 5 } & Coefficient & \multicolumn{1}{c}{$\mathrm{P}$} & Coefficient & \multicolumn{1}{c}{$\mathrm{P}$} \\
\hline Outlets (1 mile buffer) & 0.01 & 0.037 & -0.01 & 0.075 \\
Age (years) & & & & \\
18-20 & -0.94 & 0.029 & -0.70 & 0.306 \\
21-25 & 0.00 & $\mathrm{NA}$ & 0.00 & $\mathrm{NA}$ \\
26-30 & 0.52 & 0.141 & 0.30 & 0.633 \\
31-40 & 0.32 & 0.249 & -0.11 & 0.838 \\
41-50 & -0.11 & 0.682 & -0.44 & 0.356 \\
51-60 & -0.55 & 0.035 & -0.68 & 0.157 \\
61-65 & -0.61 & 0.038 & -1.05 & 0.068 \\
Gender & & & & \\
Female & 0.00 & $\mathrm{NA}$ & 0.00 & $\mathrm{NA}$ \\
Male & 0.61 & $<0.001$ & 0.58 & $<0.001$ \\
Race/ethnicity & & & & \\
White (non-Hispanic) & 0.00 & $\mathrm{NA}$ & 0.00 & $\mathrm{NA}$ \\
Black (non-Hispanic) & -0.79 & $<0.001$ & -0.45 & 0.010 \\
Hispanic & -0.51 & 0.103 & -0.78 & $<0.001$ \\
Asian (non-Hispanic) & 0.43 & 0.602 & -0.62 & 0.111 \\
Other race & & & & \\
(non-Hispanic) & -0.09 & 0.860 & -0.74 & 0.089 \\
Household income & & & & \\
<US\$ 15,000 & 0.14 & 0.494 & 0.27 & 0.395 \\
US\$ 15,000 - 25,000 & 0.00 & $\mathrm{NA}$ & 0.00 & $\mathrm{NA}$ \\
US\$ 25,000 - 50,000 & 0.54 & 0.004 & 1.05 & $<0.001$ \\
US\$ 50,000 - 75,000 & 0.97 & $<0.001$ & 1.20 & 0.001 \\
>US\$ 75,000 & 1.09 & $<0.001$ & 1.35 & 0.001 \\
Constant & 0.34 & 0.244 & 0.50 & 0.221 \\
\hline NA & & & &
\end{tabular}

$\mathrm{NA}=$ not applicable
$74 \%$ in Los Angeles. The adjusted MOR was 1.32 $(\mathrm{P}=0.24)$ for Louisiana. In Los Angeles, however, the tract-level variance was zero after adjusting for covariates. This means that there was no longer any variation in drinking status after adjusting for covariates.

The corresponding random intercept logistic regressions in Table 3 show that after adjustment for individual-level covariates there was not a strong relationship between drinking status and outlet density $^{1}$. Even though the odds ratios for the number of alcohol outlets in a one mile radius were marginally statistically significant, the odds ratios themselves were very small (1.01 in Louisiana and 0.99 in Los Angeles). There was no evidence of lack of fit based on the Hosmer Lemeshow goodness-of-fit tests (Louisiana, $\chi 2=5.56,8 \mathrm{df}, \mathrm{P}=0.70$; Los Angeles $\chi 2=2.94,8 \mathrm{df}, \mathrm{P}=0.938)$. The pseudo $\mathrm{R}$ squared values for the models were $14.0 \%$ and $10.7 \%$, respectively.

Daily ethanol consumption among drinkers: unadjusted results

Table 4 shows the relationship between the number of alcohol outlets and the quantity of alcohol consumption among drinkers. Coefficients can be interpreted as the percent increase in ethanol consumption for one additional outlet and for a $10 \%$ increase in outlets. The increases in ethanol consumption for the $10 \%$ increases in outlets are comparable across buffers of different sizes. The increases in ethanol consumption for one additional outlet are not comparable because larger buffers contain far more outlets. In Louisiana, the density of outlets was associated with ethanol consumption. The largest effect size was seen with the 1-mile buffer; the coefficient here indicates that for each additional alcohol outlet within one mile a respondent's residence the quantity of alcohol consumed over the

\footnotetext{
${ }^{1}$ Unadjusted log ORs from the model with number of outlets as the only covariate were $0.002(\mathrm{P}=0.65)$ for Louisiana and $0.21(\mathrm{P}<$ 0.001) for Los Angeles.
} 
Table 4. Percent increase in daily ethanol consumption (90 day measure and 12 months measure) for (a) one additional alcohol outlet and (b) a 10\% increase in the number of alcohol outlets based on unadjusted regressions for Louisiana and Los Angeles. For the distance measure: $10 \%$ decrease in distance to the closest alcohol outlet.

\begin{tabular}{|c|c|c|c|c|c|c|c|c|c|c|c|c|}
\hline \multirow[b]{3}{*}{$\begin{array}{l}\text { No. of } \\
\text { outlets in }\end{array}$} & \multicolumn{6}{|c|}{ Louisiana } & \multicolumn{6}{|c|}{ Los Angeles } \\
\hline & \multicolumn{3}{|c|}{ Ethanol (90 days) } & \multicolumn{3}{|c|}{ Ethanol (12 months) } & \multicolumn{3}{|c|}{ Ethanol (90 days) } & \multicolumn{3}{|c|}{ Ethanol (12 months) } \\
\hline & $\begin{array}{l}\text { Increase } \\
\text { per } \\
\text { outlet }\end{array}$ & $\begin{array}{l}10 \% \\
\text { increase in } \\
\text { number of } \\
\text { outlets }\end{array}$ & $\mathrm{P}$ & $\begin{array}{l}\text { Increase } \\
\text { per } \\
\text { outlet }\end{array}$ & $\begin{array}{l}10 \% \\
\text { increase in } \\
\text { number of } \\
\text { outlets }\end{array}$ & $\mathrm{P}$ & $\begin{array}{l}\text { Increase } \\
\text { per } \\
\text { outlet }\end{array}$ & $\begin{array}{l}10 \% \\
\text { increase in } \\
\text { number of } \\
\text { outlets }\end{array}$ & $\mathrm{P}$ & $\begin{array}{l}\text { Increase } \\
\text { per } \\
\text { outlet }\end{array}$ & $\begin{array}{l}10 \% \\
\text { increase in } \\
\text { number of } \\
\text { outlets }\end{array}$ & $\mathrm{P}$ \\
\hline $\begin{array}{l}0.1 \text {-mile } \\
\text { buffer }\end{array}$ & $2.7 \%$ & $0.1 \%$ & 0.841 & $3.4 \%$ & $0.1 \%$ & 0.719 & $13.7 \%$ & $0.2 \%$ & 0.256 & $9.6 \%$ & $0.2 \%$ & 0.319 \\
\hline $\begin{array}{l}0.25 \text {-mile } \\
\text { buffer }\end{array}$ & $8.6 \%$ & $1.1 \%$ & 0.022 & $7.1 \%$ & $0.9 \%$ & 0.003 & $2.5 \%$ & $1.2 \%$ & 0.576 & $-3.7 \%$ & $-0.5 \%$ & 0.284 \\
\hline $\begin{array}{l}0.5 \text {-mile } \\
\text { buffer }\end{array}$ & $2.6 \%$ & $1.4 \%$ & 0.019 & $2.5 \%$ & $1.3 \%$ & 0.001 & $-0.7 \%$ & $1.3 \%$ & 0.668 & $-2.2 \%$ & $-1.2 \%$ & 0.066 \\
\hline $\begin{array}{l}1.0 \text {-mile } \\
\text { buffer }\end{array}$ & $0.7 \%$ & $0.8 \%$ & 0.033 & $0.9 \%$ & $1.0 \%$ & $<0.001$ & $-0.1 \%$ & $-7.2 \%$ & 0.828 & $-0.4 \%$ & $-0.8 \%$ & 0.277 \\
\hline $\begin{array}{l}\text { Census } \\
\text { tract }\end{array}$ & $1.8 \%$ & $0.6 \%$ & 0.549 & $-0.8 \%$ & $-0.3 \%$ & 0.757 & $-2.9 \%$ & $-0.2 \%$ & 0.425 & $5.4 \%$ & $1.8 \%$ & 0.067 \\
\hline $\begin{array}{l}\text { Distance } \\
\text { to closest } \\
\text { outlet } \\
\text { (miles) }\end{array}$ & $3.4 \%$ & $0.2 \%$ & 0.337 & $1.3 \%$ & $0.1 \%$ & 0.651 & $-5.0 \%$ & $-0.1 \%$ & 0.615 & $-6.0 \%$ & $-0.3 \%$ & 0.453 \\
\hline
\end{tabular}

previous 12 months increased by $0.9 \%$. The number of alcohol outlets in the census tract and the distance to the closest outlet were not significantly associated with consumption. Based on these results we chose the 1-mile radius for the regressions below. No estimate was significant for Los Angeles, indicating no relationship between alcohol outlet density and the quantity of consumption.

Spatial autocorrelation and intraclass cluster correlation

There was no evidence of spatial autocorrelation for any outcome for both Los Angeles and Louisiana. Even though different starting values were explored, the restricted maximum likelihood (REML) estimates of the range were estimated to be zero implying no spatial autocorrelation.

The intraclass correlations in Los Angeles for the 12-month ethanol consumption was 0.008 $(\mathrm{P}=0.30)$ and for the 90 -day ethanol consumption was $0.049(\mathrm{P}=0.03)$. In Louisiana the intraclass correlation for the 12 -month ethanol consumption was $0.029(\mathrm{P}=0.06)$ and for the 90-day ethanol consumption $0.0(\mathrm{P}=0.88)$. Intraclass correlation here refers to correlation of ethanol consumption within clusters defined by census tracts. The largest and only significant correlation was for the 90 day measure in Los Angeles. We constructed a random intercept model for this outcome adjusted for the covariates as below, and found that the random intercept model is statistically insignificant from a linear regression model based on the corresponding likelihood ratio test $(\mathrm{P}=0.24)$. We therefore simply ran linear regression models on the log transformed responses going forward.

Daily ethanol consumption among drinkers: adjusted results

The average predicted values based on the Gaussian regressions of ethanol consumption of drinkers are shown in Table 5. For each categorical variable we computed average predicted values for each level of that variable. For the number of outlets variable we computed average predictions for six 
Table 5. Model-based predictions of daily ethanol consumption (ounces) of respondents who drink for both measures of consumption (12-month measure and 90-day measure) by site (Louisiana and Los Angeles). The $25^{\text {th }}$ ( $75^{\text {th }}$ ) percentile of the number of alcohol outlets correspond to 6 (27) outlets in Louisiana and 10 (28) outlets in Los Angeles.

\begin{tabular}{|c|c|c|c|c|c|c|c|c|}
\hline & \multicolumn{4}{|c|}{ Louisiana } & \multicolumn{4}{|c|}{ Los Angeles } \\
\hline & \multicolumn{2}{|c|}{ 90-day question } & \multicolumn{2}{|c|}{ 12-month question } & \multicolumn{2}{|c|}{ 90-day question } & \multicolumn{2}{|c|}{ 12-month question } \\
\hline & $\begin{array}{c}\text { Daily ethanol } \\
(\mathrm{oz})\end{array}$ & $\mathrm{P}$ & $\begin{array}{l}\text { Daily ethanol } \\
(\mathrm{oz})\end{array}$ & $\mathrm{P}$ & $\begin{array}{c}\text { Daily ethanol } \\
(\mathrm{oz})\end{array}$ & $\mathrm{P}$ & $\begin{array}{l}\text { Daily ethanol } \\
(\mathrm{oz})\end{array}$ & $\mathrm{P}$ \\
\hline \multicolumn{9}{|l|}{ Outlets (1-mile buffer) } \\
\hline 6 outlets $\left(25^{\text {th }}\right.$ percentile $)$ & 0.072 & 0.035 & 0.133 & 0.001 & 0.061 & 0.290 & 0.131 & 0.960 \\
\hline 25 outlets $\left(75^{\text {th }}\right.$ percentile $)$ & 0.084 & & 0.156 & & 0.055 & & 0.131 & \\
\hline \multicolumn{9}{|l|}{ Age (years) } \\
\hline $18-20$ & 0.039 & 0.140 & 0.105 & 0.230 & 0.054 & 0.780 & 0.128 & 0.570 \\
\hline $21-25$ & 0.098 & NA & 0.171 & NA & 0.063 & NA & 0.162 & NA \\
\hline $26-30$ & 0.083 & 0.690 & 0.198 & 0.590 & 0.047 & 0.360 & 0.140 & 0.500 \\
\hline $31-40$ & 0.086 & 0.730 & 0.154 & 0.640 & 0.056 & 0.700 & 0.142 & 0.480 \\
\hline $41-50$ & 0.091 & 0.840 & 0.185 & 0.720 & 0.070 & 0.710 & 0.147 & 0.610 \\
\hline $51-60$ & 0.070 & 0.350 & 0.115 & 0.077 & 0.054 & 0.560 & 0.104 & 0.025 \\
\hline $61-65$ & 0.074 & 0.490 & 0.119 & 0.150 & 0.056 & 0.730 & 0.113 & 0.110 \\
\hline \multicolumn{9}{|l|}{ Race/ethnicity } \\
\hline White (non-Hispanic) & 0.106 & NA & 0.186 & NA & 0.082 & NA & 0.170 & NA \\
\hline Black (non-Hispanic) & 0.056 & 0.002 & 0.105 & $<0.001$ & 0.063 & 0.250 & 0.116 & 0.012 \\
\hline Hispanic & 0.048 & 0.050 & 0.111 & 0.038 & 0.035 & $<0.001$ & 0.101 & $<0.001$ \\
\hline Asian (non-Hispanic) & 0.022 & 0.029 & 0.088 & 0.110 & 0.045 & 0.078 & 0.099 & 0.019 \\
\hline Other race (non-Hispanic) & 0.049 & 0.180 & 0.135 & 0.390 & 0.044 & 0.180 & 0.120 & 0.200 \\
\hline \multicolumn{9}{|l|}{ Gender } \\
\hline Female & 0.051 & NA & 0.103 & NA & 0.038 & NA & 0.094 & NA \\
\hline Male & 0.172 & $<0.001$ & 0.270 & $<0.001$ & 0.096 & $<0.001$ & 0.201 & $<0.001$ \\
\hline \multicolumn{9}{|l|}{ Household income } \\
\hline$<$ US\$ 15,000 & 0.073 & NA & 0.160 & NA & 0.035 & NA & 0.121 & NA \\
\hline US\$ $15,000-25,000$ & 0.101 & 0.320 & 0.187 & 0.420 & 0.037 & 0.830 & 0.099 & 0.280 \\
\hline US $\$ 25,000-50,000$ & 0.073 & 1.000 & 0.130 & 0.230 & 0.062 & 0.022 & 0.136 & 0.490 \\
\hline US\$ $50,000-75,000$ & 0.079 & 0.790 & 0.150 & 0.740 & 0.060 & 0.067 & 0.107 & 0.550 \\
\hline$>$ US\$ 75,000 & 0.087 & 0.510 & 0.141 & 0.490 & 0.083 & 0.001 & 0.171 & 0.064 \\
\hline
\end{tabular}

$\mathrm{NA}=$ not applicable

outlets and for 25 outlets corresponding to the $25^{\text {th }}$ and $75^{\text {th }}$ percentiles of its distribution.

In Louisiana, the number of outlets was significantly associated $(\mathrm{P}<0.001)$ with the 12 -month ethanol consumption measure and for the 90-day ethanol consumption measure $(\mathrm{P}=0.035)$. The counterfactual simulation shows that increasing the number of outlets from the $25^{\text {th }}$ percentile to the $75^{\text {th }}$ percentile is associated with an increase of ethanol consumption of about $17 \%$ for both the 12-month (from 0.133 to 0.156 ) and the 90-day measurement (from 0.072 to 0.084 ). This increase in exposure corresponds to an additional 14 drinks per year for each drinker in Louisiana. The percent increase from the $25^{\text {th }}$ to the $75^{\text {th }}$ percentile is consistent with the increase per additional outlet from the unadjusted results in Table 4.

For Los Angeles the coefficients for the number of outlets are not significant and the coefficients are much smaller. The regressions explain between $10 \%$ and $15 \%$ of the variation.

\section{Discussion}

Using individual level data analysis we found an association between alcohol outlet density and consumption after controlling for demographics among drinkers in Louisiana. However, we found no such association in Los Angeles. Density could be associated with consumption through the frequency of exposure to cues relating to alcohol, including the presence of alcohol itself. The design of Louisiana 
neighbourhoods may differ from Los Angeles neighbourhoods significantly, and given the same alcohol outlets densities, could still yield considerably different exposures to outlets.

The models for Los Angeles also tended to explain less variation. One possible explanation is that the association between availability and consumption is more complex in Los Angeles. Residents of Los Angeles may drive more than residents in Louisiana and access to alcohol may be less affected by the number of outlets in their neighbourhood.

Our findings add to the inconsistencies in the literature on the association between alcohol availability and consumption. Using a random intercept model for neighbourhoods, Pollack et al. (2005) did not find any association between alcohol availability and heavy drinking. The analysis might have had reduced power as both the outcome and the availability measured (outlet density, nearest distance to outlet, 0.5-mile buffer) were dichotomized. Using individual level and census tract data in California, Truong and Sturm (2007) found intraclass cluster correlations of less than $5 \%$ for dichotomous measures of heavy drinking and decided to use logistic regression rather than hierarchical models. They found that off-sale outlets were not related to problem drinking. Using individual and county level data in California, Gruenewald et al. (2002) found that restaurant densities were directly related to greater drinking frequencies and drinking after driving, whereas bar densities were inversely related to drinking after driving.

We found that the association between alcohol density and consumption is stronger when using buffers around individuals than either distance to the closest outlet or the number of outlets in the census tract. Specifically, the results suggest that the association is larger for 1-mile buffers than for smaller buffers. This is consistent with the findings by Scribner et al. (2000). Using individual level and census tract data in New Orleans, Scribner et al. (2000) found that neighbourhood alcohol availability predicted alcohol consumption better than individual availability. This finding suggests that densi- ty of alcohol outlets is a better measure of alcohol availability than distance to the nearest alcohol outlet.

We considered outlet density at various fixed distances. One could argue that outlet density should be a nonlinear function of the distance of each outlet to a given respondent. We did not explicitly consider this because of the danger to introduce too many tuning parameters as the nonlinear function would have to be estimated from the data. Because we considered multiple fixed distances such a nonlinear distance approach is unlikely to lead to different conclusions.

This study is not without limitations. First, this is a cross-sectional study and while associations found provide important clues, they are not necessarily causal. It is possible that there are more alcohol outlets near persons who drink more to meet the increased demand by those drinkers.

Second, ethanol consumption is self-reported rather than objectively measured and different alcohol drinks may have different measurement errors. While not perfect, there is some evidence (Gruenewald and Johnson, 2006), however, that a self-reported quantity-frequency measure based on the previous month of drinking exhibits good reliability.

Third, survey non-response potentially introduced bias. The danger of non-response bias is somewhat mitigated because non-response here is mostly due to non-contact rather than due to non-cooperation which can be more selective. For establishing associations (rather than prevalences) the bias is also mitigated through the presence of regression covariates. We also re-ran the analyses with weights adjusting to the current population survey (CPS) demographics and came to the same conclusions.

Fourth, the list of households from the commercial vendor within tract is not complete. The problem is somewhat mitigated because of the geographically stratified design.

Fifth, we focused on overall alcohol consumption rather than specific consumption patterns. We believe both approaches are needed and complimentary to each other. 
Sixth, our study is limited to off-premise alcohol sales. If one were to include on-premise alcohol sales conclusions may change.

In summary, findings illustrate important limitations in determining exposures to alcohol based upon close proximity and outlet density. The main purpose was to assess the magnitude of the association between exposure and drinking, so that policyrelevant solutions can be identified that can reduce the negative consequences of alcohol use. Varying geographies and travel patterns make this investigation very complex, and ultimately, more sophisticated technologies that objectively measures exposures based upon actual interactions will be needed. Furthermore, other exposures that potentially influence drinking including print ads, billboards, and television viewing where the alcohol industry expends a significant amount of resources need to be considered. Developing a model that would take the full scope of exposures into account will be helpful in isolating the most significant exposures that lead to alcohol consumption and alcohol-related morbidity and mortality.

\section{Acknowledgements}

This research was supported by grant R01AA013749 of the National Institute on Alcohol Abuse and Alcoholism. The views presented in this paper are those of the authors and do not represent those of the funding agencies.

\section{References}

Abbey A, Scott O, Oliansky D, Quinn B, Andreski PM, 1990. Subjective, social, and physical availability. II. Their simultaneous effects on alcohol consumption. Int J Addict 25, 1011-1023.

The American Association for Public Opinion Research (AAPOR), 2006. Standard definitions: final dispositions of case codes and outcome rates for surveys. 4th ed. Lenexa, Kansas, USA.

Ames GM, Grube JW, 1999. Alcohol availability and workplace drinking: mixed method analyses. J Stud Alcohol 60, 383-393.
Bluthenthal RN, Cohen DA, Farley TA, Scribner R, Beighley C, Schonlau M, Robinson P L, 2008. Alcohol availability and neighbourhood characteristics in Los Angeles, California and southern Louisiana. J Urban Health, in press. Greenland S, 2001. Ecologic versus individual-level sources of bias in ecologic estimates of contextual health effects. Int J Epidemiol 30, 1343-1350.

Gruenewald PJ, Johnson FW, 2006. The stability and reliability of self reported drinking measures. J Stud Alcohol 67, 738-745.

Gruenewald PJ, Johnson FW, Treno AJ, 2002. Outlets, drinking and driving: a multilevel analysis of availability. J Stud Alcohol 63, 460-468.

Kerr WC, Greenfield TK, Tujague J, Brown SE, 2005. A drink is a drink? Variation in the amount of alcohol contained in beer, wine and spirits drinks in a US methodological sample. Alcohol Clin Exper Res 29, 2015-2021.

Lee RE, Reese-Smith J, Regan G, Booth K, Howard H, 2003. Applying GIS technology to assess the obesogenic structure of neighbourhoods surrounding public housing developments. Med Sci Sports Exerc 35, S65.

Lipton R, Gruenewald P, 2002. The spatial dynamics of violence and alcohol outlets. J Stud Alcohol 63, 187-195.

Merlo J, Chaix B, Ohlsson H, Beckman A, Johnell K, Hjerpe P, Rastam L, Larsen K, 2006. A brief conceptual tutorial of multilevel analysis in social epidemiology: using measures of clustering in multilevel logistic regression to investigate contextual phenomena. J Epidemiol Comm Health 60, 290-297.

Pollack CE, Cubbin C, Ahn D, Winkelman C, 2005. Neighbourhood deprivation and alcohol consumption: does the availability of alcohol play a role? Int J Epidemiol 34, 772-780.

Pregibon D, 1980. Goodness of link tests for generalized linear models. Appl Stat 29, 15-24.

Robinson WS, 1950. Ecological correlations and the behavior of individuals. Am Sociol Rev 15, 351-357.

Rubin D, 1987. Multiple imputation for nonresponse in surveys. Wiley, New York, USA.

Schafer JL, 1997. Analysis of incomplete multivariate data. Chapman and Hall, New York, USA.

Scott M, Cohen DA, Schonlau M, Farley A, Bluthenthal R, 2008. Alcohol and tobacco marketing: evaluating compliance with outdoor advertising guidelines. Am J Prev Med 35, 203-209. 
Scribner RA, Cohen DA, Fisher W, 2000. Evidence of a structural effect for alcohol outlet density: a multilevel analysis. Alcohol Clin Exp Res 24, 188-195.

Scribner RA, Cohen DA, Kaplan S, Allen SH, 1999. Alcohol availability and homicide in New Orleans: conceptual considerations for small area analysis of the effect of alcohol outlet density. J Stud Alcohol 60, 310-316.

Scribner RA, MacKinnon DP, Dwyer JH, 1994. Alcohol outlet density and motor vehicle crashes in Los Angeles county cities. J Stud Alcohol 55, 447-453.

Scribner RA, MacKinnon DP, Dwyer JH, 1995. The risk of assaultive violence and alcohol availability in Los Angeles county. Am J Public Health 85, 335-340.

Theall KP, Scribner R, Cohen DA, Bluthenthal RN, Schonlau M, Farley TA, 2009. Social capital and the neighbourhood alcohol environment. Health Place 15, 323-332.

Treno AJ, Grube JW, Martin SE, 2003. Alcohol availability as a predictor of youth drinking and driving: a hierarchical analysis of survey and archival data. Alcohol Clin Exp Res 27, 835-840.

Treno AJ, Gruenewald PJ, Johnson FW, 2001. Alcohol availability and injury: the role of local outlet densities. Alcohol Clin Exp Res 25, 1467-1471.

Truong KD, Sturm R, 2007. Alcohol outlets and problem drinking among adults in California. J Stud Alcohol Drugs 68, 923-933.

Weitzman ER, Folkman A, Folkman K, Wechsler H, 2003. The relationship of alcohol outlet density to heavy and frequent drinking and drinking-related problems among college students at eight universities. Health Place 9, 1-6. 